\title{
Editorial: Computerized axial tomography
}

\section{A fundamental advance in radiology}

Fundamentally a radiograph has not changed since Roentgen $x$-rayed his wife's hand in November, I 895 . Certainly $x$-ray tubes have become much more powerful; the exposure has been reduced hundreds if not thousands of times and the silver emulsion on the film or glass plate, as it was formerly, has become much more sensitive. But the spectrum from black to white has not changed; at the white end one can depict bones, at the black end air (as in lungs), with a few intermediate densities such as fat, muscle, and viscera. For these reasons radiology has been very useful in demonstrating structures throughout the osseous system and in the chest and abdomen. But certain parts of the body, such as the brain and orbital contents, could not be depicted and for many years they remained the darkest continent of the body. Only with the aid of contrast media have advances become possible.

For nearly 80 years now the world has always related $x$ rays to conventional photography with film. But almost since their discovery, $x$ rays have been known to cause certain crystals to scintillate (give off visible light). It is this important property that has now been exploited. A few years ago, Mr. Godfrey Hounsfield, a research engineer at E.M.I. (Electrical and Musical Industries) had the idea of substituting crystals for film for the detection of $x$ rays. As his researches developed he found that he could best apply the method to the brain, which is relatively immobile compared with the thorax and abdomen, an essential feature when an $x$-ray exposure of about 4 minutes is necessary. Scanning the patient's head with a narrow beam of $x$ rays and a crystal detector enables a computer to extract the maximum amount of information from the readings. This produces a picture in which the grey levels are calculated to a degree of accuracy roo times greater than in conventional $x$-ray tomography. In I97 I a prototype machine was installed at St. George's Hospital where Mr. Hounsfield worked in co-operation with the neuroradiologist, Dr. James Ambrose; the immediate dramatic results exceeded all expectations. In the very first case examined a frontal lobe glioma, as well as the ventricles, was readily revealed. In April, 1972, they gave their first account of their results in about seventy cases. World-wide interest was immediately aroused amongst neuroradiologists and four machines are now installed in the United Kingdom, about fourteen in the United States, and two on the continent of Europe.

The interest to ophthalmologists lies in the fact that the orbit as well as the brain can be examined by transverse axial tomography because there is a good differentiation of tissue density in the orbit. It was previously mute to $x$ rays but can now be very satisfactorily examined, as the following paper shows. It is perhaps appropriate to sketch briefly the history of radiology of the orbit and reassess it in the light of this new development. During the first world war, Sir James Mackenzie Davidson, a London ophthalmologist and radiologist, developed a precision technique for the localization of foreign bodies in the eye. Radiological advances thereafter were few and far between until contrast methods began to be developed some 30 years ago. Of these, orbital phlebography is probably the most 
important and rewarding, though it has severe limitations in displaying tumours, but it is safe and easily accomplished in the majority of cases. Carotid arteriography, with a view to outlining the ophthalmic vessels, also has a limited place, but radiological examination of the orbital contents has until now been very unsatisfactory. Two other techniques have been tried but both have severe disadvantages. One, which consists of injecting air into the retrobulbar space, was applied as early as 1927 . It is an unpleasant procedure for the patient and, although it is probably not dangerous, the yield of positive information regarding space-occupying masses has been very limited. Another technique consists of injecting a water-soluble contrast medium. This is probably more rewarding diagnostically, but can be hazardous. Sometimes the contrast substance seeps backwards in between the layers of the dura mater and this can lead to dangerous complications. Even without this complication nausea and vomiting, spasm of the central retinal artery, and even blindness have been reported.

The introduction of the E.M.I. scanner promises a new and above all safe method of investigation. 